\title{
Early Greek Astrophysics: The Foundations of Modern Science and Technology
}

\author{
Xenophon Moussas \\ The Observatory of the National and Kapodistrian University of Athens, \\ Astrophysics Laboratory, Faculty of Physics, \\ Panepistimiopolis, GR 15783 Zographos, Athens, Greece
}

Received 2013-10-19; Revised 2013-12-09; Accepted 2014-02-04

\begin{abstract}
The foundations of modern science and technology, metrology, experimental physics, theoretical physics, theoretical mathematics, astrophysics, including applied optics and spectroscopy go back to the Prehistoric, Presocratic, Classical and Hellenistic Greece. This tradition is well deep rooted in time, going back mainly to the epoch of the prehistoric Aegean sea, the mainland of Greece and the Islands, Cycladic, Euboea, as well as Crete, at the late Neolithic period, probably starting back at 4400 BC. Humans observe the sky before the prehistoric era and wonder and as even Plato declares, these observations that lead us to try and understand the Cosmos make us humans as well, since the actual meaning and etymology of this term human in Greek, the word ANTHROPOS, is the one that looks up, observes the ordered Universe and tries to understand its nature and its hidden Laws which govern it. Science and Philosophy emerged as a result of the human efforts to live even more successfully within a hostile environment. Humanity eventually manages to understand Nature and especially the Cosmos, using the Pythagorean principle: "Nature can only be understood accurately by the use of Mathematics, which expresses the underlying Laws of Nature and which explain all natural phenomena based on the Principe of Causality.
\end{abstract}

Keywords: Ancient Astronomy, Ancient Astrophysics, Ancient Physics, Prehistoric Science

\section{INTRODUCTION}

Philosophy, Science and Technology are continuously and radically changing human life, especially during the last four to six thousand years and very actively during the last two hundred years or so. All of us today take for granted all the daily applications of technology, new and old, like our computers, the spacecraft technology, the telescopes which search the sky using the whole spectrum of the electromagnetic radiation and all other applications. Our lives now depend enormously on Technology, which is based on many scientific applications, unknown to the lay person. How did all this happen? How was Science and Philosophy created and especially Astronomy and Astrophysics? How did we succeeded to go to the Moon?

To give a quick flashback we should go back to mythical figures, such as Dionysus, Hephaestus,
Prometheus and Orpheus and to Giants of Thought, such as Thales, Plato and Archimedes, as we all stand on their shoulders, according to the comment of the Great Newton.

Prehistoric people observe with great interest the movements of the celestial bodies and gradually discover the Harmony of the Cosmos (Fig. 1 and 2). They observe and eventually record the movements of the Sun and the Moon, the perpetual circular motion of the stars, the changing seasons, the eccentric interesting Dance of the Planets, with their forward and backward movements which make our brain to wonder and think.

The movements of celestial bodies, their daily, annual, monthly, as well as their much longer periods, are the subject of study for people and inevitably lead to the development of useful calendars and out of these, to the development of Mathematics and Astronomy. 


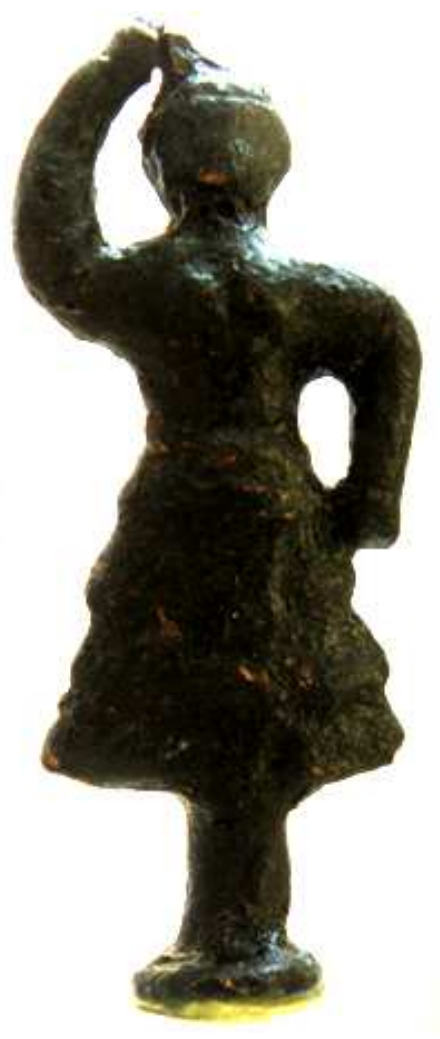

Fig. 1. Figurine of the sacred peak of the small island of Cythera depicts a man observing, probably the Sun at sunrise from the peak of the Mountain (St. George). The Archaeological Museum of Piraeus

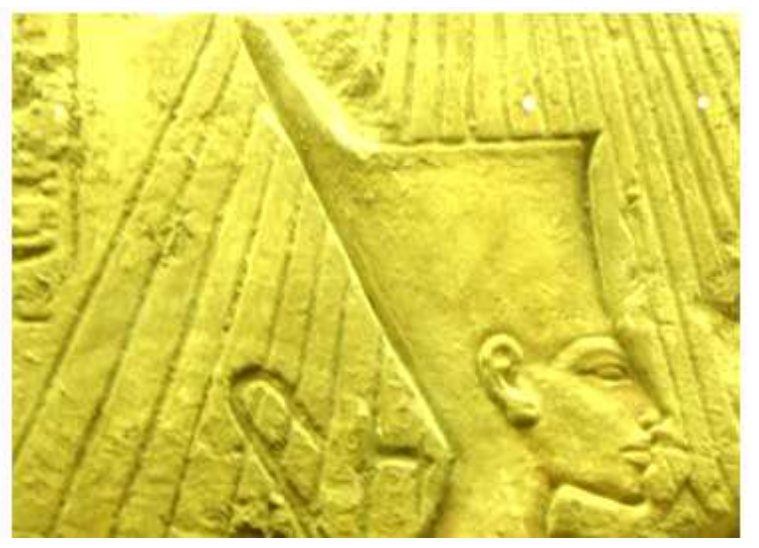

Fig. 2. The measurement of the inclination of the rays of the Sun, in some place on Earth and at noon, enables compliance with the solar calendar. On the wonderful Egyptian relief discerning the Pharaoh counting the height of the sun at midday and determine the start of the year, or the date in general
We have shown recently (Tsikritsis et al., 2013), that there exist clear indications of complex and complicated calendars, dating back to the fifth millennium BC, which are based on periodicities of the Sun and the Moon and on the, totally unexpectedly, periodicities of the planets Venus, Jupiter and Mars, which are related to the human activities, not only the agricultural ones, as didctated by the solar year, but also to the cycles of human pregnancy, that is the period of nine lunar months, the 8 year periodicity of the planet Venus, as well as other totally unexpected finds on terracotta vessels, dating almost at the middle of the 5th millennium BC.

Stars and astronomy lead the travelers and all marine nations, in antiquity. Since the roads of antiquity in Europe are the marine roads and in particular the Mediterranean shipping routes, a region which is the birth and the begin of the European and the Western civilization, where the roads of mechanitise and travel are exclusively the marine roads. The marine nations, predominantly the Greek nation, in the passing of many Astronomy is essential for the trade, for finding new resources, such as copper and tin and new regions or countries appropriate for the trading or the establishment of colonies. Along with the Technology and the arts practices and techniques, which allows to built better ships, which acquire additional stability, speed and endurance, are developed gradually and sciences and philosophy (Neugebauer, 1975).

The technology was quite advanced by the time of the Trojan War and certainly well before this. The Greeks have advanced their Technology for the construction of weapons and ships, as well as the related techniques. One such feature we encounter in Homer, where the Great Poet informs us about a ship that decides automatically how it goes to its destination, depending upon the direction of the wind that automatically changes the direction of sail.

Mythology reflects the Knowledge developed by ancient gods or semigods and heroes, which have contributed generally to Knowledge, Science and Philosophy, such as Dionysus (Berthelot, 1888), of Hephaestus (in Metallurgy), of Prometheus and Orpheus (in Astronomy).

Science, Culture and Philosophy are universal. They belong to all nations. The Greek contribution to these is essential, because the foundations of every scientific discipline are set in Greece.

The written form of Orphic hymns (see ancient text in Orphic Hymns) was noticed by a group of ancient literary philologists, under the guidance of Onomacritus (560-490 BC), who was given the task of collecting and recording the Homeric Epics under the auspices of Hipparchus (not the astronomer), archon of Athens (527- 
514 BC), a mammoth project started earlier by Pisistratus. The Orphic Hymns provide us with important information about the notion of Causality and the notion about the Laws of nature. In one of these poems we encounter the term Law (of Nature) which is used in the same sense as today: "celestial law that puts the stars in position and motion"(Fig. 3 and 4$)$.

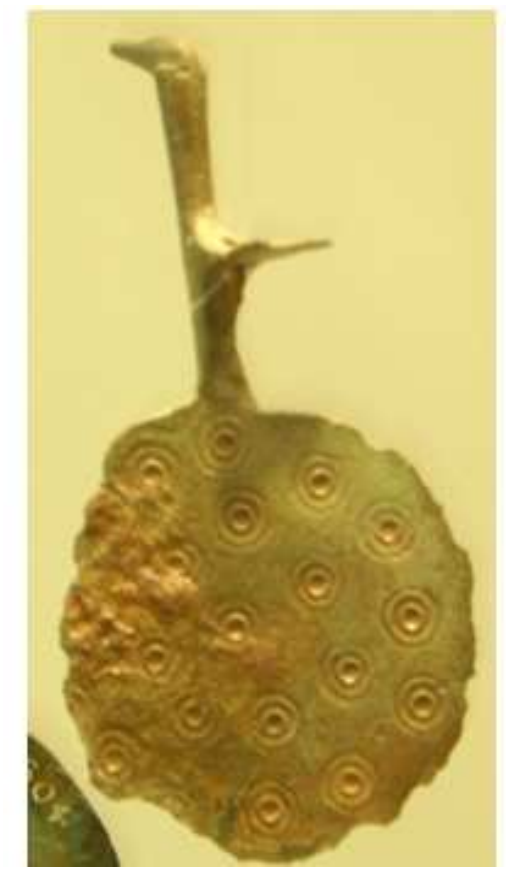

Fig. 3. Votive, 8th century BCE. Schematic map of the sky? National Archaeological Museum, Athens

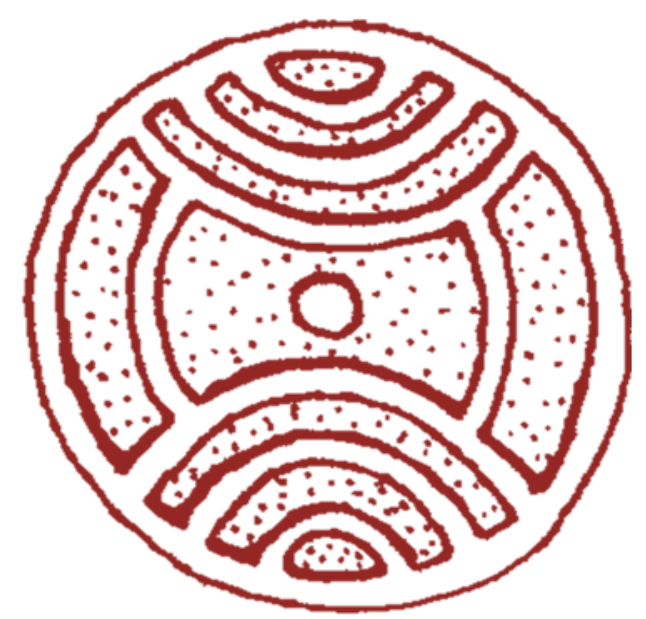

Fig. 4. Seal, the 7th century BCE-like display of the celestial sphere
At the same era the Metallurgic industry, the invention of the wheel, the wheel of the Potters and the lathe radically, change the course of humanity through the development and use of Technology.

Orpheus is certainly one of the most important mythical figures who contributed to the development of Philosophy and especially of Natural Philosophy. According to our knowledge, Orpheus was born on the Mount Olympus in Pieria, in Macedonia, Greece, probably before the Argonauts and before the end of the Trojan War, according to the ancient sources.

We can speculate that he existed as a real person and also that his grave was located for centuries in the Leibithra (Olympus), where he lived. Travellers describe his tomb centuries later. Therefore Orpheus is a legendary figure who lived, worked and influenced profoundly Greece, Humanity and Culture. He taught Musaeus, the first astronomer in the Mythology, who made the first celestial sphere used by the Argonauts and Jason, in order to find their way to the Black Sea, by solving, perhaps in some way and for the first time, the difficult problem of calculating the longitude on a travelling ship. It is interesting to note that Isaac Newton later called the problem of longitude as the "problem of the Black Sea", a term that has survived since then in the international literature. The Orphic Hymns prove that the roots of Science goe back in time for millennia, as first discovered and proved by the late Chasapis and his student Papathanasiou (1978), which continued his work and whose studies are used in this chapter. In the Orphic hymns we can read the origins of Science, not only for Greece, but for all humanity. Certainly all the civilizations had developed the Practical Astronomy and transacted some Mathematical knowledge.

\section{ARCHAIOASTRONOMY}

Astronomy as a practice started almost at every length and breadth on Earth, while all the temples, even in areas where the sky is covered with clouds for the most days of the year, are oriented according to a clear, or frequently hidden, astronomical orientation, which helps humans to keep time, to obtain a form of a clock and, most importantly, a calendar ensuring that the agricultural activities are performed on time. The use of Astronomy is imposed everywhere on Earth. The ancient temples, as well as other public buildings in Greece and in other countries, exhibit a special orientation, in order to keep a calendar properly, with significant periods the 
ones of sowing and of harvesting, accompanied by customs, such as pollinators events on plowing and sowing the fields, some of which are kept even today in Greece and other countries, like the festivity of St. Demetrius, on October 26, a festivity which probably replaced the festivity of Demeter, the Goddess of Agriculture and the Cereals. It is worth mentioning that in ancient Egypt we encounter a similar orientation of the temples around October 22, which is called "achet" and which corresponds to the seeding. At this date, as found in the Temple of Abu Simbel, the sunrays at sunrise illuminate the statues of the God Amun Ra, the Sun and King Hey Orakti, while the beginning of the year was set to coincide with the flooding of the lifegiving Nile, marked by the heliacal rising of the brightest star in the sky of Egypt at sunrise, the star of Sirius.

Similar guidelines are followed also in Greece, where the ancient temples and some churches today, are facing towards East, so that the sunrays at sunrise on the Saint's day enter and illuminate the icon of the Saint, while some cities are oriented toward the East during the solstices. Alexandria is oriented position rises the Canopus, the second brightest star in the sky after Sirius, on July 20th the birthday of Alexander the Great.

In Egypt and in Greece, the main axis of the city and of the large churches, are often aligned with the sunrise at some important day for keeping properly the accurate calendars.

Astronomy as a Science, perhaps even from the prehistoric times, separated from its practical value and significance and became an independent Scientific branch, even more theoretical with the passage of Time and based on accurate mathematical Methods. Of course, the scientists of these eras had the interest to know when and where the eclipses of the Sun and Moon will take place, not only because people are impressed by them and act with fear in eclipses' presence, but also for reasons of prestige.

Similarly, these scientists wanted to understand the movements of the planets and even to predict them, although these movements are of higher complexity and are difficult to be predicted. The construction of lunisolar calendars and the prediction of the celestial phenomena eventually led inexorably to our today's Technology and Science. The development of the calendars and the representation of the motion of the celestial bodies, especially the planetary motions, in order to predict the eclipses, requires the development of complex Mathematics, a fact that led ultimately to the formulation of the Laws of Astronomy and Physics.

\section{CALENDARS BASED ON THE SUN AND THE MOON AND THEIR ROLE IN CIVILIZATION}

The calendars have played a very important role for the development of civilization, because they require a good knowledge of Mathematics and Astronomy, for enabling humans to regulate properly their life's, their agricultural activities. The most advanced calendars are usually based on the movements of the two dominant celestial bodies, the Sun and the Moon, the points of sunrise and sunset on the horizon during the year and the height (angular distance) from the horizon at noon. The lunar month, which changes slightly over time, has an average duration of a synodical lunar month of 29.530589 days and the year, that lasts a little less than 365 days and $1 / 4$ of the day, are the protagonists in the evolution of Astronomy and Mathematics very early in the History of mankind. From various indications we are certain that people are measuring these periodicities and are finding the multiples of these abovementioned periodicities, in which the phases of the Moon are repeated in multiples of the solar (tropical) year.

Noticing that the 19 year period of Meton, or the enneakaidekaetiris, which according to the sources was discovered by Meton in his observatory of the Pnyx (an observatory right behind the podium of the Athenian parliament) in $432 \mathrm{BCE}$, is equal to 235 synodic months, allows to predict the time, when the Moon shall reappear with the same phase (e.g., the New Moon, or the first quarter, or the Full Moon) in the same part of the sky (relative to the background of the stars), since this phenomenon exhibits a periodicity of 19 years. The Metonic period, in some Greek calendars, consists of 110 months of 29 days and 125 months of 30 days, or a total of 6940 days, or 235 synodic months, with a deviation of $7.5 \mathrm{~h}$ and gives an error in the length of a year of approximately $7 \mathrm{~min}$. The Callippic period of 76 years was also used in some long calendars, additionally to the Metonic cycle, although it is not more accurate, as it gives a larger error for the year.

Using the periodicities of Saros, which are approximately equal to 18 years, 11 days and $8 \mathrm{~h}$, or 6585.3213 days and exactly equal to 223 synodic months, which is about 242 drakonic months (within $51 \mathrm{~min}$ ) and approximately equal to 239 anomalistic months, the period which is useful for predicting the Lunar and the Solar eclipse,, phenomena always impressing the people since prehistoric times. Even more accurate is the threefold periodicity, called as 
Exeligmos (54 years and one month or 19,756 days), which indicate seven better the place on Earth, form where the eclipse is visible.

The complex astronomical instrument of the second century BC we call "Mechanism of Antikythera", a mechanism which is also a complex astronomical computer, incoroporates the keeping and the usage of various kinds of calendars, a solar and two luni-solar calendars of 19 years, 76 years and 18 years and 54 years, which are based on the phases of the Moon and the luni-solar eclipses, as we have previously described The study of the oldest scientific device and computer has been made me a series of studies by (Svoronos; 1903; 1908; De Solla, 1955; Price, 1974; Rados, 1905; 1910; Rediadis, 1903; Rehm, 1907). Theofanidis (1934) and especially and by (Wright et al., 1995; Wright, 2002; 2003; 2005a; 2005b; 2005c; 2006a; 2006b) and with much better techniques and results by (Freeth et al., 2006; 2008; Freeth, 2009; Moussas, 2011, Bromley, 1986; 1990a; 1990b; 1990c; Devevey et al., 2008; 2009; Henriksson, 2009; 2013; Moussas et al., 2007; Gourtsoyannis, 2010; Marchant, 2008; Malzbender et al., 2001; Chondros, 2009) that revealed several important aspects of this complex ancient instrument with unexpected results.

\section{THE GREEK MIRACLE}

We call as the "Greek Miracle" the birth and development of Philosophy and Science in Greece. People wonder how and why these started in Greece. The combination of the prerequisites for the development of Science would expect us to believe that this could happen at all longitudes and latitudes, at all Civilizations on Earth and that Science could have been born by all people. But this did not happened. Certainly, all Cultures have developed Practical Astronomy, Elementary arithmetic and Calendars, to some extent and in a larger or smaller degree. But science was born only in Greece and this constitutes the "Greek Revolution", the "Greek miracle".

We present the thesis that Science and Philosophy developed as a result of the habit the Greeks had developed, the unique habit to express themselves in public, at the Agora, that is the parliament, a habit that historically has only been possible in Greece, where we encounter the high diversity of the small city-states, of the small communities, where almost every person has the opportunity to express himself in public and state his opinion based on his arguments.
The oldest Parliament of the World is known to be located at Poliochni, a small prehistoric town of the island of Lemnos. In this parliament we see stone seats of the 'MPs', of the advisers, of the Lord Prime Minister, within a small room. The "Greek miracle", the birth of Science and Philosophy, may be the result of the birth of Democracy, which allows the expression of the personal opinion and which was permissible and possible occasionally in the small city-states of Greece and not in large states, such as in Egypt, in Persia or China. The exercise of the citizens in expressing themselves led to the formulation of the Laws of Nature and the birth of Science in Greece. The Greece, along with its biodiversity and "ideopoikilotita" (ideodiversity), has led to the development of new ideas and gradually to the development of the Philosophy and of the Sciences (Moussas, 2011).

But why and how did Science appeared in Greece? It is the geographical position of Greece probably which allowed societies to develop, at a time when the rest of Europe was frozen, it is the diversity of the soil, with many valleys and islands, which allowed, or probably, to state it more correctly, led to the development of many societies, which may also have been completely different in their structure and attitudes, as it is illustrated within the differences between the society of Athens and of Sparta. The diverse soil has led to the rich biodiversity in Greece. For example, Cyprus or Crete host much more plants in number than Britain. In Greece, the numerous islands offered safe areas, that is areas without the presence of great beasts, because these beasts could not survive, as they needed more space for their existence. It is worth noting that in the Greek islands we encounter remains of pygmy elephants, dwarf hippopotamuses and that some of such animals have survived until our epoch, such as the unique small horses of the Greek island of Skyros.

The variety of the terrain and the isolation of one geographic region to another, in Greece, leads to the production of a variety of ideas and concepts, a variety of Cultures and Ideas. Greece has always been a fountain of new Ideas, simultaneously a think-kit and think tank. "Greece can do very well culture" said some years ago President Mitterrand and this applies in perpetuity. The exchange of ideas is the Agora, the place where the citizens gather to talk and to exchange ideas, which is at the same time the center, the heart of the city-state, where, as the name already suggests, a place where Ideas are exchanged and questioned. In large countries, like in Egypt or Persia, is impossible to allow a type of an Agora, where everyone can present his opinion, where 
all have the right to express themselves, because in populous countries, if all were allowed to express themselves, it would not ultimately be possible for a decision to be taken. The luxury of the direct democracy is a luxury of the small states and of cities, if not all and not for all seasons. The crowd often takes wrong decisions on many crucial issues, as in the case of the misjudgements in the trials of the renownphilosophers, like Socrates and Aristarchus, which are uproarious examples of tragic decisions. But the important thing is that the Agora established a tradition for Dialogue, for questioning, which ultimately leads to development of Dialectics. Through this development humans understand determinism and eventually perceive that there are Laws of Physics that govern Nature. With these Laws we can interpret the natural phenomena, predict some of them, such as the eclipses of the Sun and the Moon, to understand the climate, to try to predict the weather based on the seasons. All these interpretations and predictions drastically affect their lives and lead us to live them even better.

\section{IDEOPOIKILOTITA OR IDEODIVERSITY}

In Greece, Astronomy, from practical discipline becomes a theoretical Science, which uses theoretical Mathematics, with mathematical proofs, which enable the opportunity of conducting better predictions and it is in Greece that Astrophysics is born. Astrophysics, the branch of Physics that tries to explain the cosmic phenomena and discuses the nature of the astronomical objects, based on theoretical Thought and Reason rather than the fear of the unknown, a fear that generates all prejudices we find in all the pre-scientific societies. The unscientific prejudices afflict the world, until the Greek philosophers demolish the illogical beliefs and this eventually leads to the Greek Scientific Revolution and the "Greek miracle". The branch of Logic, with the aid of Mathematics and Rseason, dissolves gradually with its light the dark clouds of prejudice, which unfortunately and even today finds suitable conditions and reconcentrates by covering and distorting reality, by torturing people and nations.

The exercise of citizens to express themselves appreciately led gradually and inexorably to the formulation of the Laws of Nature and the birth of Science in Greece. Greece, along with its large biodiversity, exhibits also ideopoikilotita i.e., a wide variety of ideas and concepts, which led to the development of new ideas and to the development of
Philosophy and of the Sciences. Indeed, professor Haida Liang of the Nottingham Trent University in England, Chinese in origin, informed me, by commenting on my theory, that in China Philosophy and the development of new ideas happened in the hisrorical period when China existed in the form of many small states, in which the civilization developed (Moussas, 2011).

\section{OBSERVING THE SKY}

As I say every year in the course Astrophysics and Astronomy to the students at the Department of Physics and the Department of Mathematics, of the National and Kapodistrian University of Athens, there was a time when we had no television and people at night could observe, when the sky was clear, the circular motion of the stars and of the other astronomical objects as well, thus they gradually learned to study the peculiar motion of the planets, they studied the cosmic rhythms, the "Music of the Spheres" which Pythagoras noticed, the rythic rate of the appearances of the phases of the Moon, the New Moon, the first quarter and the Full moon, the daily circular motion of the Sun and the spiral motion of the Sun during the course of one year, the gradually changing positions of sunrise and sunset on the horizon, throughout the year, which were associated with the transitions the seasons, of the cold and the hot seasons and the variation of the length of the day and of the night. Humans begin to realize that the change of the seasons, of the day and night, their successive order of appearance is regulated by the apparent movement of the Sun, which affects directly and indirectly their lives.

\section{THE FIRST ACCURATE ASTRONOMICAL MEASUREMENTS}

Humans develop calendars based on the Sun and the Moon, that is calendars which become very complicated, as they want to take into account the phases of the Moon combined together with the yearly motion of the Sun. Recently we discovered (Tsikritsis et al., 2013) that in all terracotta vessels, called as "teganoschema", or "frying pans" due to their shape, are inscriptions that can be interpreted as numbers in a very primitive simple form of writing. These numbers (which have the form of single dots, or of triads) in the majority of teganoschema give periodicities which are related to the period of menstruation, the period of pregnancy of the women and the period of revolution of Venus around the Sun, as observed with respect to Earth, together with an eight 
year period, which is called in Greek "Octaetiris", which is a period of 99 lunar months, or two Olympiads, or two "tetraetiris". This period was attributed to Cleostratos, from the Aegean island of Tenedos, but our finds show that this period is marked in the teganoschema millennia before, in the islands of the Aegean sea, which include Crete and the Greek mainland, starting back at $4400 \mathrm{BCE}$. In some teganoschema we read the periods of the planet Jupiter and in other teganoschema the period of the planet Mars. If our finds are correct, then this proves that humans established careful observations of the planets in the 5 th millennium BCE, which means that they established records of the positions of the planets, with their retrograde motions, for many years in order to know periodicities which last for hundreds of days and with accuracy. It also proves that humans developed the ability to measure accurately and to derive mean values of physical quantities.

\section{THE FIRST PHYSICS EXPERIMENTS}

At Hellenistc times, the period after Alexander the Great, the motto of ancient astronomers in Greece, in their struggle to reproduce the past and predict the future positions of the Sun, the Moon and planets was the following: "Save the phenomena", that is to reproduce the motions, even if they do not understand dedcompletely the physical mechanism behind these phenomena. These Hellenistic astronomers, of course, followed the much older tradition of Pythagoras and his disciples, which tried to understand the so called "Music of the Spheres" and interpret the physical phenomena, that is the idea and practice of modelling based on the Laws of Physics (Fig. 5 and 6).

Pythagoras first called the universe Cosmos because of its beauty (Placita philosophorum 886.B.5) and its order and harmony. The symmetries and the periodicities the Pythagoreans had noticed led to the creation of Science, through the concept of Causation and Determinism, the existence of Laws of Nature which are expressed accurately only with the use of Mathematics. In fact, it is worth noting that when the Greeks use the term Mathematician, they mean what we call today in modern terms as a Theoretical Physicist.

The representation and the prediction of the position of celestial bodies and particularly of the planets and of the eclipses, is of particular significance in Human History and especially in Greek History and the History of Philosophy and Science.

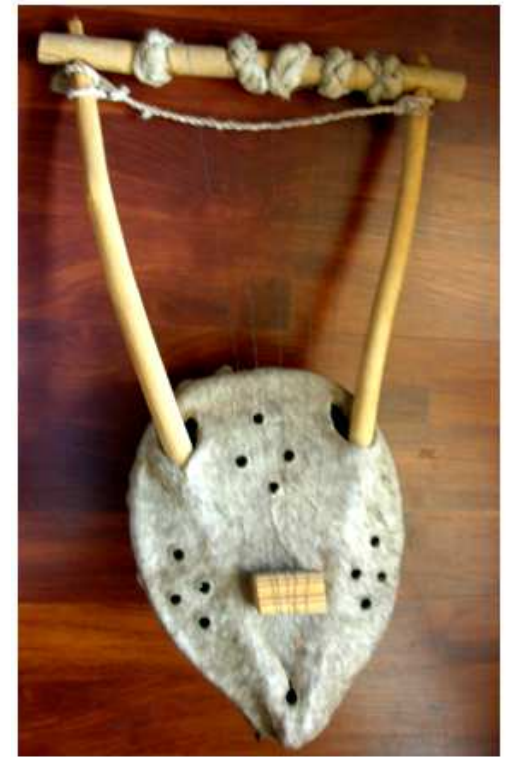

Fig. 5. Contemporary simple lyre of Africa turtle (Greek cheloneion), leather and strings, that probably lead Pythagoras to perform the first experiments in Physics, by conducting measurements

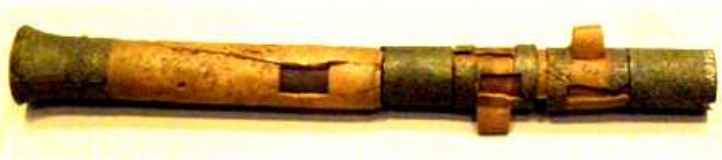

Fig. 6. Music and harmony played an important role in the development of physics, experimental and theoretical as Pythagoras performed the first experiments with measurements that lead him to establish the dependence of the frequencies played to the tension and length of the chord and eventually to understand of the important role of mathematics to the expression of laws of nature a accurately; Part of delicate bone flute with a complex system of regulating holes which allow a better operation of the musical instrument. 2nd CBC. National Archaeological Museum, Athens

The legendary story of iron hammering, that Pythagoras heard outside a blacksmith's workshop and which probably lead him to conduct the first designed experiment, accompanied with measurements, in Physics. The story says that Pythagoras was walking out of a blacksmith workshop and heard the master with its apprentices to forge anvil. Pythagoras finds paradoxically that they sound not as a cacophony, but that this forging has a pleasant rhythm and sounds like music. He enters the blacksmith's workshop and finds 
that the hammers have very specific weights, physically tangible, with very specific mass ratios and so produce sounds like Music. He conducts experiments with chords under tension and understands that the frequency produced by chords depends on the length of the chord and the force that stretches it.

Pythagoras inexorably is driven to the musical octave. Thus, he finds the correspondence between the musical notes and the condition of the string which vibrates. The great astronomer' uses a similar scale to study the Harmony of the Spheres, in the movements of the planets around the Sun (Papaspirou, 2013). The harmonic Titius Bode law on the distances of the planets, which applies also to the satellites of Jupiter, of Saturn, is the result of harmoniously arranged bodies that essentially follow the same principles as the strings, with the planets, or their satellites, to be in resonances, dancing to the sound of the secular Music of the Spheres.

Astronomers and even Medical doctors, from the time of Pythagoras until the time of Kepler, use the logic the harmonious music of the spheres even to cure their patients.

\section{HARMONIC ANALYSIS AND MODELLING}

The Greeks used extensively Mathematical models, i.e., the Laws of Physics, as they perceive them during each period of the historical evolution of their Civilization, with different types and different forms. These models can be compared with the modern mathematical methods of Spherical Harmonics, introduced in 1782 by Pierre-Simon de Laplace in his book "Mécanique Céleste" and used since then in numerous applications, e.g., by the great Gauss and other important Physicists. The Greek models exhibit closer similarities with the Fourier series. I know that this comparison is unexpected and astonishing, but I find it valid, as well as historically necessary and instructive. The motions of the planets, as perceived by the ancient observers and as viewed from the Earth, are complex. These observers discovered that the planets change their longitude in the sky, as well as their speed, when at a particular moment and during their motion on the night sly, they start to go backwards, in relation to the fixed background of the stars, for a shorter period of time. The planets perform this retrograde motion, going back and then forth again, for a longer period. The planets do not move randomly, but within the context of the "cosmic dance", they dance according to the "Music of the Spheres" of Pythagoras. The Greek Mathematicians and Astronomers, in their effort to reproduce the motion of planets, introduce multiple concentric spheres, a sphere for each planet and reproduce some of the movements of the Earth around its axis, that is the diurnal motion of the sky, the motion of the Earth around the Sun, which introduces the retrograde motion of the planets and a sphere that reproduces the movement of the planet around the Sun and one off the ecliptic plane, that is around the plane on which the Earth moves around the Sun.

Eudoxus of Rhodes introduces 27 spheres that reproduce the motions of all the visible by nacked eye planets, of the Sun and of the Moon, as seen from Earth, while Callippus improves these motions with a few additional spheres, increasing their number to 34 , while Aristotle uses 56 spheres, so that there are no logical gaps in the movements of the planets and the transmission of their motions.

Alternatively, the Astronomers of the time develop models with eccentric circles, models with circles and epicycles and model which use equants and which resemble the Fourier series. In this way they reproduce in an improved manner the motion of the planets, as seen from Earth and with thesemodel of epicycles or eccentric circles manage to explain the change in the luminosity of the planets, which has been already observed. Astronomers of the Islamic and Arabic Civilization add more epicycles, thus making the model more realistic and this astronomical tradition is followed by the Byzantine Astronomers and then by the Astronomers of the Western European Civilization.

Even Kepler, the Giant of Physics who revolutionized Astronomy many centuries later, by the introduction of his three Laws of planetary motion and as the figure who introduces ellipses, follows the Greek Tradition. The ellipses, or more generally the conic sections, have been invented and studied by the Greeks before the time of Euclid. Kepler uses in his first work, the "Mysterium Cosmographicum:, the mathematical technique of epicycles in order to produce the motion of the planets. It is a fact that the epicycles are simpler and more easily reproduced than the ellipses, when we come at the stage of performing astrothetical calculations. Euclid presents the ellipses which are studied theoretically by Apollonius of Perga.

The epicycles and the epicyclic motions have been introduced for the motion of the planets by the mathematician and astronomer Apollonius of Perga, who invented the epicycles in order to reproduce realistically the motion of the planets as seen from Earth, basically by 
performing a change of coordinate systems, namely the Sun -centered system of reference and the Earthcentered system of reference. Mind due that Apollonius, the one who studies extensively the conic sections, does not use them for the motion of the planets, because the circles can be defined and reproduced very easily and even more important, if we operate with one epicycle which revolves with the double frequency of the deferent circle, the resulting motion almost coincides with an ellipse. Additionally, circles are very useful for mechanical calculations as well, as they can be used to construct automata that perform mathematical calculations automatically, as we have in the case of the Antikythera Mechanism.

Hipparchus refined the epicyclic models based on accurate measurements he conducted in Rhodes and almost two centuries later Ptolemy uses the equant. Certainly his work is based on more measurements, while Ptolemy further promotes models of epicycles, which stay in use much longer than one could imagine, long after Kepler's laws have been accepted by the scientific community.

\section{THE ASTROPHYSICS OF ORPHEUS}

Orpheus in his hymns (poems) postulates that Nature consists of soil, sea, air (ether) and Uranus (the celestial bodies). In the Orphic hymns and for the first time, the nature of celestial bodies is discussed, presented and analyzed in terms of the Nature and the natural phenomena (Fig. 7). For Heaven Orpheus uses the term "presvygenethlos", the "first to be born", so that according to the Oprhic description of the Universe it is accepted that the Universe has a beginning. Orpeheus and his followers, state that the Universe, that is the Cosmos, is untamable and endless (without end), Nature is apator and autopator, i.e., Nature is born without a father, or that Natureis born by itself. Nature incloses Light everywhere, Nature is the mother of everything (Papathanasiou, 1978).

The universe is "pyripnoun", that is full of fire, it is constantly moving and the sky is only a part of the World that we see and not the whole Universe. The Cosmos is "Krototeknos", which means that the Universe gave birth to Saturn (and to Time). The Cosmos moves spirally around the Earth. Orpheus, like Homer, distinguishes the planets from the stars, on their motions. This tradition of Natural Philosophy presented in the Orphic teaching and texts probably started much earlier, long before man started to develop writing.

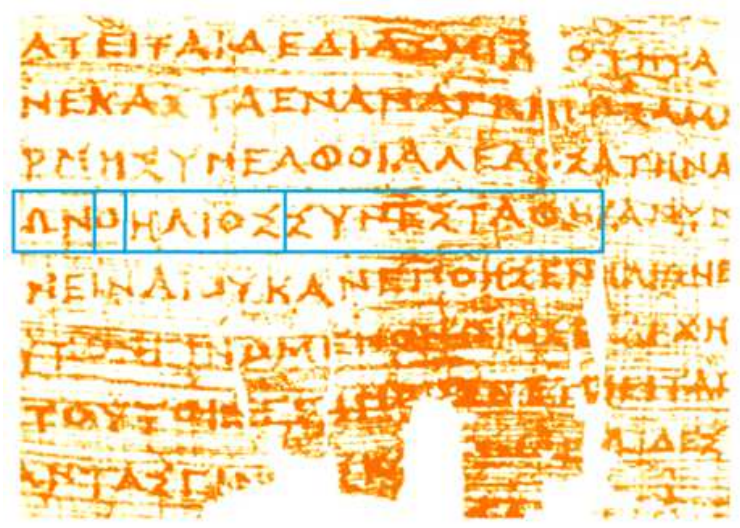

Fig. 7. A small fragment from the Orphic Hymns on the nature of the Sun: ... of which the Sun is composed of ... [ $\Omega \mathrm{N}$ $\mathrm{O} \mathrm{H} \Lambda \mathrm{IO} \Sigma \Sigma \mathrm{YNE} \Sigma \mathrm{TA \Theta H}]$

Astronomy has very deep roots within the Greek civilization. The astronomy of Minos in Crete, which has been accidentally discovered, as hidden within the Minoan palaces and the tops of the mountains of Crete and Cythera, in Holy Summit show that the "Music of the Spheres" is already recorded by the constructors and architects of the palaces and the other building structures of these eras.

\section{PAPYRUS OF DERVENI AT MACEDONIA, GREECE}

The oldest known text written on papyrus or any perishable material, Europe's oldest surviving manuscript, is the so called Papyrus of Derveni, where Derveni is a small village in Macedonia, Greece. The papyrus was discovered in a $4^{\text {th }}$ century $\mathrm{BC}$ tomb ( 340 to $320 \mathrm{BC}$ ), just before the time of Alexander the Great and it reflects knowledge of Astrophysics. It looks like the Orphic hymns and philosophically it sounds like the presocratic philosophers Anaxagoras, Leucippus and Democritus and Diogenes of Apollonia, while as possible authors of this papyrus many names have been suggested, such as the Euthyphron of Prospalta, Diagoras of Melos and Stesimbrotus of Thasos. The existence of this text shows that humans at that time had a great interest to eschatological and astronomical issues (Betegh, 2004).

\section{THE GREEK PHILOSOPHERS ON ASTROPHYSICS}

It is worth looking at the view of the Greek philosophers and especially of the Ionians and their view 
about the construction of Cosmos, that is the Universe, about the stars and the planets and that these views are very close to those of modern Physics and Astrophysics. In fact, it is easy to identify the striking similarities, the almost identical perceptions of ancient philosophers, like Leucippus and Democritus, with the contemporary perception. Here, we list some of them succinctly.

According to Leucippus, the Universe was created and with it a swirl of atoms, which revolve around its center. The heavier atoms move towards the center. Initially, the Universe acts as fluid and as it rotates, it heats up, becomes very hot and creates the matter, out which the stars are made. Here we observe that Leucippus uses common knowledge, since if you rub a stick rotationally, then you have production of heat and light. Leucippus concludes, therefore, that in the Universe the process of friction heats the celestial bodies and produces energy, the energy of the Universe, the energy of the stars and of the Sun.

The stars, according to Leucippus and Democritus, are created by grouping of atoms. The stars light up because of their extremely fast rotational speed. The Sun, similarly, becomes hot by this rotation mechanism and the same applies for the stars, which become luminous by their fast rotation.

The Earth was created by a vortex, where atoms gathered and created mass, which that rotates and heats up and finally gets cooled. The light atoms stay up in the air, while the heavy atoms strive towards the center, in order to form water and earth.

Leucippus and Democritus, as well as other philosophers, claim that the eclipses of the Sun are rarely visible, unlike the eclipses of the Moon, because their diameters are unequal. The Worlds (the Universes or Solar Systems) are infinite in number, are distributed in space and are made out of an infinite number of atoms. Some worlds (that is, planetary systems) have Suns (stars) and some have not. Our Sun is a star.

The sight, according to Leucippus, is the result of the images of the objects which fall on the pupil of the eye.

On the number of Worlds ("Cosmoi") Thales believes that there exists only one world, Democritus, Epicurus and his student Metrodorus of Lampsacus, as well as Seleucus believe in the existence of an infinite number of Worlds (Placita philosophorum 886.B.8),

The Ancient Greek philosophers argue about the shape of the Cosmos. All proposed shapes are at least axially symmetric. The Epicureans and the Stoics believe that the Cosmos is of spheroid shape or (some of them) of conical shape (which might mean pineal too), or it is egg shaped (Placita philosophorum 886.C.12).
What governs the universe? The Ionian philosophers wonder and in summary the answer is that the governing principle of the Universe is the Soul (Psyche), which also means spirit, accompanied together with Provision, or Providence, Precaution or Forethought, that establish order in the Cosmos.

Democritus, Epicurus and all the Atomic Philosophers disagree and suggest that the vacuum, empty space, is not governed by the action of the Psyche, or anything similar, while Aristotle rejects all these opinions, while he dictates that the celestial bodies communicate with each other (Placita philosophorum 886.D.4).

The Philosophers of the Greek tradition seek to find answers to the question of whether the Universe is perishable or not. Do we have preservation of mater? The Cosmos is indestructible, according to Pythagoras and to Plato, while the Stoics believe that the Universe is created by God and it is perishable, because it is made out of matter. Epicurus considers that the World was born and it is corruptible, like an animal or a plant, while Xenophanes considers the Cosmos as being unborn, eternal and imperishable.

Which elements constructed the universe? Physicists (that is, the Physical or Natural Philosophers) suggest that earth is the basic element as Earth is the center of Cosmos. Pythagoras believes that the a ether, or the fifth element, is the first in quality and succession. Empedocles considers a ether as the first element, then as second comes the fire, then follows the water and after this the earth. The Sun is made of fire, which, if concentrated, produced other fires like the ones we find on Earth. Plato believed that the visible world was made as an Exemplar of the mentally, intelligibly World, the Nous of the Demiourge of Cosmos and that matter originates out of fire and earth, initially and of water and air after, while Pythagoras teaches that the world is made of five solids, which he calls mathematical. According to this tradition, the earth is made from particles that have the shape of the hexahedron (the cube), the fire is made from tertahedra (pyramids), air is made out of octahedra, water from icosahedra and from the dodecahedra the sphere of the Cosmos. Plato follows the Pythagoreans in this doctrine (Placita philosophorum 887.A.7).

In fact, Plato introduces what we could call "Plato's elementary particles", or "Plato's quarks", which are just two right triangles, out of which all the Platonic solids, as we call them, although their origin is Pythagorean, are constructed. These two triangles are: (1) an equilateral orthogonal triangle with the two equal sides of one unit and (1) a right triangle, with one side equal to unity and its hypotenuse equal to two units (Fig. 8). 


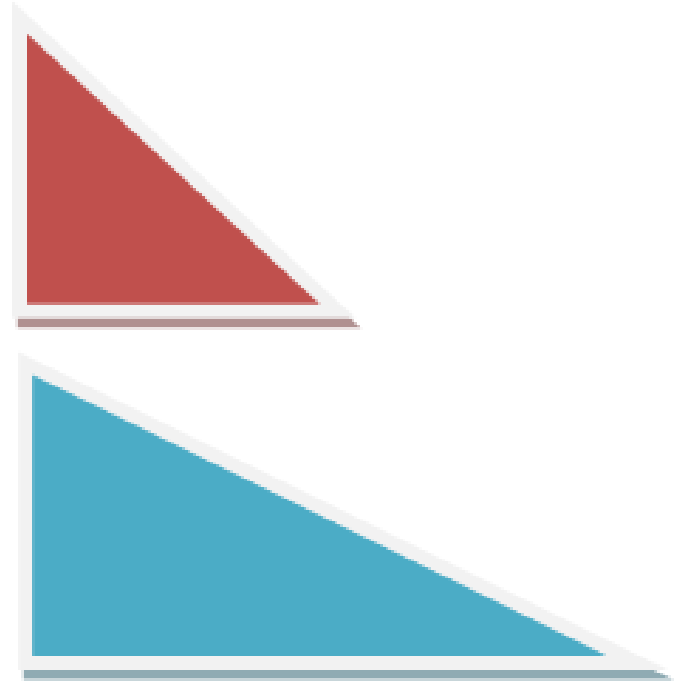

Fig. 8. The tow Pythagorean triangles of Plato. The Cosmos is made of these two components, that are Plato's elementary particles, or quarks

Parmenides inform us that the Cosmos was made out of entangled vortices, other sensed and other rarefied, other bright and other dark.

Epicurus teaches that the limits of the Worlds are dense for some and rarefied for other and that some Worlds are moving, while other Worlds are standing.

What exists beyond Cosmos? Some, like Pythagoras, suggest it is empty space, while the Stoics teach that within "ekpyrosis", the infinite is diluted (Placitaphilosophorum 887.F.4). The theory of "Ekpyrosis" is one of the precursor theories of the Big Bang Theory, according to which the Big Bang is always followed by the Big Crunch, in which the Cosmos is destroyed in order to be reborn again with the advent of a new Big Bang and this procedure takes place when all celestial bodies, the ones characterized by periodic motions, return to their initial positions.

Philosophers in antiquity developed different perceptions of the Milky Way. Some of them believed that the Galaxy is a burned star. Various quotations give the impression that they believed that the Galaxy is composed by the remains of a burned comet (named Phaethon by the Greeks), a comet that passed close to Earth.

Leucippus and Democritus understood that the Galaxy consists of many stars that we cannot distinguish and in this the Galaxy is similar to matter, in which we cannot distinguish the tiny atoms it is made of, although they are distinct in nature, as also distinct are the stars in the Galaxy, which again are indistinguishable.

\section{THE IMPORTANCE OF COMET AND METEORITE OBSERVATIONS FOR THE BEGINING OF ASTROPHYSICS}

The cometary and meteorite observations in all Civilizations and especially by the Greeks, have been extremely important for the development of Astrophysics and Physics in general and especially for Philosophy. The reason is very simple. Over millennia comets have been passing very close to the Earth and our human ancestors had the opportunity to observe them better than we can today with the use of large telescopes. Observing the comets gives us several clues in order to infer many conclusions about their nature and then to generalize for the case of the other celestial bodies. Complementary observations came from falling meteorites, some of which were associated to comet, that is the cometary debris.

The main result of the cometary observations is that the stars are conglomerations, concentrations of hot gases.

In Greece we encounter the myth of Phaethon, a myth appearing in various versions and which was very popular. From this myth we know that he was a comet that has passed very close to the Earth and it is certain that several fragments of it fell on surface of the Earth. During these passages it was possible to see the streams of gases and dust ejected from the cometary nucleus due to its proximity to the Earth.

The Greek astronomers saw the jets and assumed further that the stars are made of gasses, concentrated as they state and that some stars have openings, resembling the bellows of a blacksmith's workshop, out of which gasses are emitted. The cometary debris and the meteorites permitted humans to conclude that celestial bodies are very hot, possibly made of the same materials as the Earth and in some cases even to conclude that the Sun is a huge hot stone, which is certainly larger than the peninsula of Peloponese and which has an almost round shape.

Anaxagoras must have observed the comet of Halley, out off which a small part created the fall of the meteorite at Aegospotami in the Hellespont and simultaneously created the initiation of Science and Natural Philosophy.

Anaxagoras is said to have predicted the fall of the meteorite at the Aegospotami. This could probably be done either on the basis of the correlation, which is certainly made since the prehistoric times, between the comets and the fall of meteorites and because he observed a bright comet, which we know today that it 
was the comet of Halley and he assumed, rightly, that a meteorite fall was possible, either because the Greek astronomers knew already from very ancient observations the periodicity of this comet, so that you may need to rename it as the comet of Anaxagoras and expected, as it has also happened in the past, the falling of meteorites and of numerous meteors. Modern writers refuse to justify, which is according to my opinion unfounded, the possibility that Anaxagoras had predicted the fall of the meteorite.

Our information is supplemented by Aristotle, hundreds of years after the fall of the meteorite, which writes in his Meteorological that this rock fell during the day and that it is related to the fall of many shooting stars, while at the same time a very bright and large comet appeared in the western sky.

Plutarch informs us that Anaxagoras had predicted that celestial bodies could fall to Earth and the Roman philosopher Pliny the Elder, in the 1st century AD, adds that the meteor of Aegospotami was a rock as large as a carriage and that it was exhibited as a tourist attraction for nearly five centuries. Pliny connects the body with a bright meteor which was visible for 75 days before the fall of the meteorite and presented calculations which indicate that Halley's Comet was visible from Greece for three months (June 4 to August 25, 466 BC). It has been argued that the comet Halley, at the time of Anaxagoras, could have upset the orbit of another body that fell to Earth (Gibbon, 2009).

The comments sections of meteorite collected Anaxagoras in conjunction with the descriptions of witnesses certainly led to the development of human perception that can interpret astronomical phenomena based on the natural born simultaneously with astrophysics as a result of these observations and thoughts Anaxagoras.

Certainly, the ancient people, for millennia happened to observe many times comets from a near distance with respect to Earth and sometimes observed these comets and meteors to fall on Earth. Thus, probably, this led to the perception that the stars are made up out of condensated gases, since they observed these comets to eject gases of volatile materials, such as water, hydrocarbons, hydrogen cyanide, ammonia, carbon monoxide and carbon dioxide and methane. Probably, the swastika is a symbol of a comet which passed very close to Earth. Ancient Chinese manuscript of the third century BC mention the swastika, because it depicts the outflow of gases, which originated the idea that the stars are made out of concentrated gases.
Certainly, the ancients must have seen this, when some of the comets came extremely close to Earth and when even some even fell on Earth, causing enormous catastrophes. This, at the same time, allowed people to make important observations on the nature of the comets and thus enabled them to gain knowledge, for generalizing conclusions about the nature of the celestial bodies.

The reference to the comet "Phaethon" allows us to conclude that the Ancient Philosophers refer to the observations of some comet with a, naturally occurring, long tail, or even to a disintegrating comet, which has been observed at a very close distance from the Earth. In both of these scenario, they would have observed very clearly volatile material, stemming from the obvious sources on the comet, that ejected steams of gases and dust particles, which enabled humans to understand the nature of this type of celestial objects and then generalize for all kinds of celestial objects, including the stars and the Sun. In modern times, astronomers have observed many comets with spacecrafts and telescopes and we established their nature by using also spectroscopic methods and in situ measurements.

Of great interest is the early understanding of the Greeksabout the comets. The Pythagoreans understand that they their appearance is periodic. Anaxagoras and Democritus believe that they are synods of stars near the Pleiades star cloud. Aristoteles proposes that they are earthly fumes of soil. Aristotle informs us very well that the comets acquire an earthy texture and that they also contain volatile materials.

Straton says that the comets are composed by starlight inserted in dense clouds, which become bright from the reflected light. He also informs us that the comets are bright because they are dense clouds illuminated externally, from the light of the Sun.

Herakleides of Pontos informs us that the comets are composed of a cloud which is illuminated by an external source. The disciples of Aristotle at the Peripatetic School also believe that the comets are made of gases and that they are composed of volatile materials.

The philosopher Epigenes says that the comets are a mixture of gases mixed with soil, that is he correctly understands the composition of the comets.

Metrodorus states that when comets fall on the Sun then they produce eruptions, which is an observation we made via the SOHO satellite only ten years ago. Xenophanes insists that the comets are systems of moving gases. 


\section{ON EARLY SPECTROSCOPY}

The great progress in Astrophysics came as a result of Spectroscopy, i.e., the use of the analysis of light and of the received electromagnetic radiation in general, in its components (that is, the various colours), or put more correctly, by the analysis of the electromagnetic radiation at the whole spectrum of wavelengths or frequencies, an analysis that permits to accurately estimate the temperature and the chemical composition of a star, or a planet and further to determine its speed, its angular velocity, as the relevant astronomical object turns around its axis, as well as a plethora of other important physical information about the studied celestial object. These analyses have their roots back to Greece, especially with the study of the rainbow, the reflection and deflection of light and other optical phenomena (Fig. 9).

Let 's look at the history of this very important domain of modern Astrophysics. The Science of Optics, including the reflection and refraction of light, already exists in the Greek literature and in several cases it is accompanied with the usage of mathematical proofs.

Damianus the Larissaian (from Larissa of Syria, the son of Heliodorus, 4th century AD), a Greek philosopher, discusses the problem of reflection on a mirror, a study included in his book on Optics. Heron, who was a Mathematician, an Engineer and a polymath, in his book about Catoptrics proves that if the angles of reflection of the light rays are equal, then the length of the ray path is minimum. (see also Damian, On Optics, Optical, 14), that is he states the principle of shortest path, also known as the Principle of Fermat, the principle of least time, according to which the light, during its path through a medium and from one point to another, follows the shortest, that is the fastest optical path, in order to move in the shortest possible time and the Law of Snell, for the case of the refraction of light.

The observations for the rainbow lead to the birth and development of Spectroscopy. From the observations of Iris (the rainbow, in Greek) they conclude that, exactly as we see the oars of a boat in the sea to appear "broken", also the light coming out of the rainbow gets reflected in the droplets of the rain which constitute it. According to their teachings, this is the reason we see the rainbow when the Sun is behind us. Then, they try to explain the colours of the rainbow as observed in the given order.

Anaximenes and Metrodorus try to explain not only the phenomenon of the rainbow, but also the various appearances of the Sun, the red colour of the sky at sunrise, the blue colour of the sky during the day.

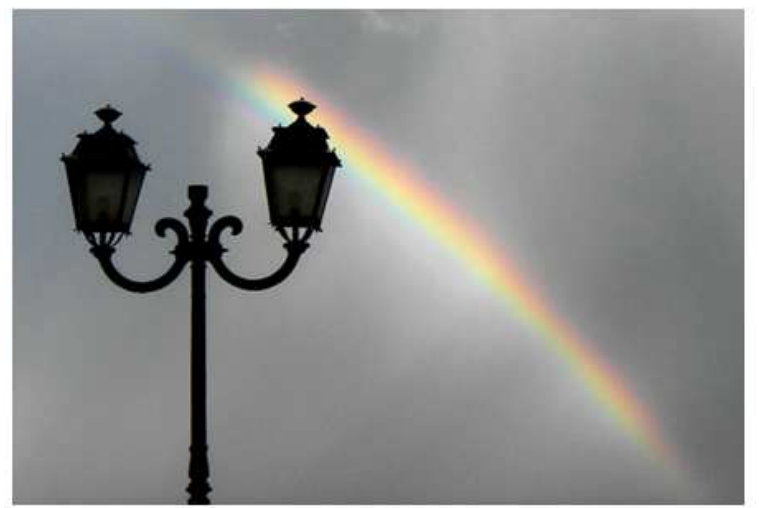

Fig. 9. The observations of rainbow and the early attempts to explain physically the mechanism by Greek philosophers lead eventually to the development of spectroscopy and understanding of the Cosmos through the analysis of EM radiation (rainbow at Corfu)

\section{AN ANCIENT MECHANICAL COSMOS, THE SO CALLED ANTIKYTHERA MECHANISM, AN EPITOME OF GREEK PHILOSOPHY}

The Antikythera Mechanism is certainly the oldest computer we know and additionally it is also the oldest complex astronomical instrument. The Antikythera Mechanism subverts everything we think that we know about the lack of interest of the Greek civilization Technology. It looks like it were off-season, while in fact it is the epitome of Greek Philosophy.

It epitomizes the Philosophy, because in order to build a Mechanical Universe, you need to assimilate Greek philosophy, the Natural Philosophy of the Ionian philosophers and you have to put it into practice.

The Antikythera Mechanism is the culmination of the teaching and understanding of the Pythagoreans, a teaching which led to the exact wording of our knowledge about the Universe, with the mathematization of our understanding about the World and about Nature. This Mathematization leads inevitably, through experimentation and observation and through the perception that Nature is harmonious, that there exists the "Music of the spheres", which reflects how Cosmos works, as found with properly designed and realized experiments like the ones performed with musical instruments by Pythagoras and measurements that led through fine analysis and inductive thinking to the formulation of the laws of physics, as we understand them today. Note that the signature of 
Pythagoras, a pentagon, is present in the device around an axis that moves the Moon around the Earth following Keplrer's second law to a good approximation, or even perhaps all the three laws.

Our modern world and today's technology is based precisely on the same Principles of Physics, the notion of Causation and Determinism and the belief into human capacity for understanding Nature, for interpreting and even repeating the studies in experiments in the laboratory natural phenomena, duplicate them and use them for science and technology. All modern technological achievements are based on the same principles underlying the Antikythera mechanism and have their background in it.

The Mechanism has been constructed by Greek scholars, probably between 150 and 100 BC. As it turns out and according to our analysis, the construction of the mechanism is based on measurements taken by Archimedes and his students, who continued to flourish after the tragic death of Archimedes at Syracuse (Stamatis, 1974). Our finds prove that Archimedes was also a Physicist and an Astronomer, who established and run at Philosophical school in Syracuse, where his students continued to perform astronomical observations, including the solar and lunar eclipses, while using clocks to measure the time of these celestial events (Moussas, 2011).

The Antikythera Mechanism is a complex analog and accurate digital computer, that works with carefully designed and manufactured gears having small teethes. The gears perform certain mathematical operations, as they move around the axes of the Mechanism. The movements of the gears move pointers giving the positions of various heavenly bodies, that is of the Sun, the Moon and possibly the visible by necked eye planets. The Mechanism is the first Mechanical Cosmos, a clockwork Universe, the first Planetarium and possibly an Astronomical clock. It is very probable that the structure was accompanied by several automata, which performed various acts, such as it is the case with the various medieval clocks and exactly as we read in many ancient books describing similar astronomical instruments. The motion of the Mechanism was probably caused by the use of weights and counterweights and a float, which resemble those of the detailed description of the clock of Archimedes, or the medieval clocks and the cuckoo clocks.

Of particular importance is the newest discovery about the motion of the Moon, since eight years ago we discovered that the lunar motion of the Mechanism followed approximately Kepler's second Law, while now it appears that with a link between two elliptic gear gives the more accurate trajectory and speed of the Moon probably follows all of Kepler's three Laws.

It is interesting that the name of this type of instruments, like the Antikythera Mechanism, as we read in ancient books is "Table", when the instrument has large dimensions, even more surprising, "Tablet", when it has small dimensions.

\section{ACKNOWLEDGEMENT}

The John F. Costopoulos Foundation is gratefully acknowledged for generous support. The Alexander Onasis foundation for helping the exhibition at the NASA J F Kennedy Space Center. The research that leads to the analysis of computer tomographs was carried out in collaboration with Prof. M. G. Edmunds, Prof. J. Seiradakis, Dr. T. Freeth, Dr. H. Mangou, Ms M. Zafeiropoulou, Mr. Y. Bitsakis, Dr. A. Tselikas and the National Archaeological Museum in Athens. Special thanks to the director Dr George Kakavas, Dr. N. Kaltsas, Ms R. Proskynitopoulou, Mr M. Makris and all the staff of the Museum, that enable us to obtain the raw data). The X-ray data were gathered by a team from XTek Systems (UK), now Metris (NL), led by Dr. R. Hadland, special thanks are due to A. Ramsey and A. Ray. We thank the team from Hewlett-Packard (US), led by Dr. T. Malzbender, who carried out the surface imaging and for using his PTM excellent method for analyzing the surfaces of bodies and software. We appreciate the support of C. Reinhart of Volume Graphics. The photos have been produced using Volume Graphics software and our X-Tek data. Thanks are due to Dr Goran Henriksson for discussions and calculation on ancient eclipses, Dr Ch. Kritzas for estimating the age of the Antikythera Mechanism based on the shape of the letters of the manual, Prof Giovanni Pastore for many discussions on the Olbia Archimedes gear, Prof. Manolis Mikrogiannakis for drawing my attention to the Homeric automata, Dr Arnold Lebeuf for discussions on Meton and Saros cycles and ancient calendars, Dr Alexandra Coucouzeli, Prof. Magda ElNoweimy and Mr. Panos Papaspirou many interesting discussions, Mr. D. Kriaris for creating several bronze models of the Antikythera Mechanism that we have used at various exhibitions around the world, Dr. F. Vafea and Prof. M. Papathanassiou for interesting long discussions on the structure of the Mechanism, about astrolabes and about ancient texts. Thanks are due to the University of Athens for support. 


\section{REFERENCES}

Berthelot, M., 1888, Collection des Anciens Alchimistes Grecs. 1st Edn., G. Steinheil, Paris.

Betegh, G., 2004. The Derveni Papyrus: Cosmology, Theology and Interpretation. 1st Edn., Cambridge University Press, Cambridge, ISBN-10: 0521047390, pp: 456.

Bromley, A.G., 1986. Notes on the antikythera mechanism. Centaurus, 29: 5-27. DOI: 10.1111/j.1600-0498.1986.tb00877.x

Bromley, A.G., 1990a. The antikythera mechanism. Horol. J., 132: 412-415.

Bromley, A.G., 1990b. The antikythera mechanism: A reconstruction. Horol. J.

Bromley, A.G., 1990c. Observations of the antikythera mechanism. Antiquar. Horol., 18: 641-652.

Chondros, T.G., 2009. The development of machine design as a science from classical times to modern era. Proceedings of the International Symposium on History of Machines and Mechanisms, (HMM' 09), Springer Netherlands, pp: 59-68. DOI: 10.1007/9781-4020-9485-9 5

De Solla, P.D.J., 1955. Clockwork before the Clock. Horol. J.

Devevey, F., A. Rousseau, C. Vernou, P. Cauderlier and C. Magister, 2008. The astral disc of chevroches. Cosmology Across Cultures.

Devevey, F., A. Rousseau, C. Vernou, P. Cauderlier and P. Causeret et al., 2009. The Zodiacal "curved disc" of Chevroches. The Antikythera Mechanism Research Project.

Freeth, T. 2009. Decoding an ancient computer. Scient. Am., 301: 76-83.

Freeth, T., A. Jones, J.M. Steele and Y. Bitsakis, 2008. Calendars with Olympiad display and eclipse prediction on the Antikythera Mechanism. Nature, 454: 614-617. DOI: 10.1038/nature07130

Freeth, T., Y. Bitsakis, X. Moussas, J.H. Seiradakis and A. Tselikas et al., 2006. Decoding the ancient greek astronomical calculator known as the antikythera mechanism. Nature, 444: 587-591. DOI: 10.1038/nature05357

Gibbon, E., 2009. The decline and Fall of the Roman Empire. 1st Edn., CRW Publishing Limited, Cirencester, United Kingdom.

Gourtsoyannis, E., 2010. Hipparchus vs. Ptolemy and the Antikythera Mechanism: Pin-Slot device models lunar motions. Adv. Space Res., 46: 540-544. DOI: 10.1016/j.asr.2009.08.030
Henriksson, G., 2009. Ten solar eclipses show that the Antikythera Mechanism was constructed for use on Sicily. Department of Physics and Astronomy, Uppsala University.

Henriksson, G., 2013. Thales of Miletus, Archimedes and the solar eclipses on the Antikythera Mechanism. Proceedings of the21th Annual Meeting European Society for Astronomy in Culture, (SAC' 13), SEAC.

Malzbender, T., D. Gelb and H. Wolters, 2001. Polynomial texture maps. Proceedings of the 28th Annual Conference on Computer Graphics and Interactive Techniques, Aug. 12-17, ACM Press, New York, USA., pp: 519-528. DOI: $10.1145 / 383259.383320$

Marchant, J., 2008. Decoding the Heavens: Solving the Mystery of the World's First Computer. 1st Edn., Windmill Books, ISBN-10: 0099519763, pp: 336.

Moussas, X., 2011. The Antikythera Mechanism. 2nd Edn., Union of Greek Physicists, Athens.

Moussas, X., J. Seiradakis, T. Freeth, M. Edmunds and Y. Bitsakis et al., 2007. Communicating Astronomy to the public. Proceedings of the 55 conference IAU Commission, (IAU' 07), Athens.

Neugebauer, O., 1975. A History of Ancient Mathematical Astronomy. 1st Edn., Springer, New York, ISBN-10: 354006995X, pp: 503.

Papaspirou, P., 2013. Greek influences to Kepler. PhD Thesis, University of Athens, Athens, Greece.

Papathanasiou, M., 1978. Cosmolocical and Cosmogonical Notions in Greece during the $2 \mathrm{dn}$ millenium BC. PhD Thesis, University of Athens, Greece.

Price, D.D.S., 1974. Gears from the Greeks: The Antikythera Mechanism-a Calendar Computer from C. 80 BC. 1st Edn., Science History Publications, New York, ISBN-10: 0882020196, pp: 70.

Rados, C., 1905. Comptes rendues. Proceedings of the International Archaeological Congress, (IAC' 05), Athens, pp: 256-258.

Rados, C., 1910. On the Antikythera Treasure, astrolabe, anaphoric clock, hodometers. Athens.

Rediadis, P.D., 1903. Der Astrolabos von Antikythera. 1st Edn., Beck and Barth, Athen, pp: 8.

Rehm, A., 1907. Philologische wochenschrijt: Cols.

Stamatis, E., 1974. Archimedes Works. 1st Edn., TEE Publishing House, Greek, Athens.

Svoronos, J.N., 1903. Die Funde von Antikythera. 1st Edn., Athen, pp: 80. 
Svoronos, J.N., 1908. Das Athener Nationalmuseum. 1st Edn., Athen.

Theofanidis, J., 1934. Sur l'instrument en cuivre, dont des fragments se trouvent au Musee Archeologique d'Athenes et qui fut retire du fond de la mer d'Anticythere en 1902. Praktika tes Akademias Athcnon, 9: 140-149.

Tsikritsis, M., E. Theodossiou, V.N. Manimanis, P. Mantarakis and D. Tsikritsis, 2013. A Minoan eclipse calculator. Mediterranean Archaeol. Archaeometry, 13: 265-276.

Wright, M.T., 2002. A planetarium display for the Antikythera mechanism. Horol. J., 144: 169-173.

Wright, M.T., 2003. Epicyclic gearing and the antikythera mechanism. Antiquar. Horol., 27: 270279.

Wright, M.T., 2005a. The Antikythera mechanism: A new gearing scheme. Bull. Scient. Instrument Soc., 85: 2-7.
Wright, M.T., 2005b. Epicyclic gearing and the Antikythera mechanism. Antiquar. Horol., 29: 5163.

Wright, M.T., 2005c. Counting months and years: The upper back dial of the Antikythera mechanism. Bull. Scient. Instrument Soc., 87: 8-13.

Wright, M.T., 2006a. The Antikythera mechanism and the early history of the moon-phase display. Antiquar. Horol., 29: 319-329.

Wright, M.T., 2006b. Understanding the Antikythera mechanism. Proceedings 2nd International Conference on Ancient Greek Technology, Technical Chamber of Greece, (TCG' 06), Athens, pp: 49-60.

Wright, M.T., A.G. Bromley and H. Magkou, 1995. Simple X-Ray tomography and the antikythera mechanism. PACT, 45: 531-543. 